Military Technical College

Kobry El-Kobba

Cairo, Egypt

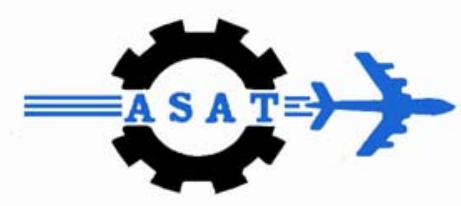

12-th International Conference

on

Aerospace Sciences \&

Aviation Technology

\title{
PARAMETERS OPTIMIZATION OF DIVERSITY ANTENNAS IN MOBILE ENVIRONMENTS
}

\author{
Mohamed A.H. Eleiwa*
}

\begin{abstract}
The optimum configuration of antenna diversity can be designed by considering the characteristics of both the antenna effective gain $\left(G_{e}\right)$ and the correlation coefficient $\left(\rho_{\mathrm{e}}\right)$. Therefore, this paper introduces a procedure to evaluate and optimize antenna diversity parameters. The proposed procedure is based on numerical calculations of relevant derived formulas for $G_{e}, \rho_{e}$, and the normalized mutual resistance $R_{12}$ between diversity antenna ports. Moreover, different co-polar, and cross-polar antenna pattern E-plane and $\mathrm{H}$-plane cuts, are used to evaluate and analyze the correlation characteristics, and hence optimum antenna diversity configuration is obtained. The developed procedures are then used to analyze parallel-dipoles space diversity antenna system.
\end{abstract}

\section{KEY WORDS}

Mobile Communications, Diversity Techniques, Antennas

\section{NTRODUCTION}


Terrestrial Wireless communications suffer from multipath, shadowing and fading effects. Of the various techniques available to combat multipath, antenna directivity is the most powerful but also the most complicated and least economical, because of the extra hardware (and power) associated with the use of more than a single antenna. This technology, which has been used mainly in large installations, such as cellular base stations, is now emerging as a viable economical solution even in handheld devices. Array processing was previously complex and expensive, but with the advent of simpler processing algorithms, simulation software and exponentially growing DSP engines, the limitations are gradually being mitigated.

Antenna-diversity techniques [1] generally fall into four categories. Spatial diversity involves the use of physically separated identical antennas. The phase centre of each antenna is also spatially separated. Pattern or beam diversity uses co-located antennas that are of different size, shape, orientation and/or material. These antennas have dissimilar radiation patterns and their signals are combined in phase due to their collocation. Polarization diversity uses two antennas oriented at $90^{\circ}$ to each other. The result is mutually orthogonal polarization states, such as horizontal and vertical; left-hand circular and right-hand circular; or $\pm 45^{\circ}$ slants. The antennas used in polarization diversity schemes are often identical. Finally, transmit/receive diversity schemes employ separate antennas for transmit and receive functions, so frequency filtering is not needed. The technique to combine the two signals which are received from the diversity scheme should be considered, and an appropriate combining technique can yield better performance. There are four general combining techniques. The maximal ratio combining (MRC) technique maximizes the signal-tonoise ratio after combining. The equal-gain combining (EGC) brings the received signals to a common phase and combines them in voltages. The selective combining (SEC) technique selects the strongest of the two received signals. The switch combining (SWC) is based on a threshold level below which the signal switches to the other antenna. The MRC, EGC, and SEC require more complex equipment, perform better, and cost more than SWC, which needs only one front end. Among the MRC, EGC, and SEC, the performance of MRC is the best, but the equipment is complicated. The performance of SEC is lower than that of MRC and EGC, but the equipment is simple to build. Although combining techniques are not within the scope of this paper, the implications for antenna design are relevant.

The diversity performance is dependent on the number of diversity branches and on the correlation coefficient between the received branches [2-4]. Each diversity scheme can reach the same performance if the branch correlation coefficients are the same. The optimum configuration of antenna diversity can be designed by considering the characteristics of both the correlation coefficient and the antenna effective gain. Therefore, this paper introduces a procedure based on numerical calculations for $G_{e}$ and $\rho_{e}$, diversity antennas ports mutual resistances measurements, and antenna patterns in different planes for copolar and cross-polar components. The proposed procedure are then applied to investigate the correlation characteristics of N/2 and short dipole antennas. Finally, space diversity antenna systems composed of two parallel dipoles with different lengths and spacing, are investigated.

\section{DERIVATION OF ANTENNA DIVERSITY PARAMETERS}


Since the optimum configuration of antenna diversity can be designed by considering the characteristics of both the correlation coefficient and the mobile antenna effective gain, so first it is necessary to establish a theoretical expression for the mobile antenna effective gain that takes into account the vertically polarized (VP) and horizontally polarized (HP) incident radio waves in multipath environments. The ratio between the mean received power of antenna over the random route $\boldsymbol{P}_{\text {rec }}$ and the total mean arriving power $\boldsymbol{P}_{\boldsymbol{V}}+\boldsymbol{P}_{\boldsymbol{H}}$ can be considered as the mean effective gain (MEG) of the mobile antenna in the environment. It is assumed that the average over a random route in an environment equals the average over the environment. This ratio is defined as the MEG of the mobile antenna in the environment and is denoted by the symbol $\boldsymbol{G}_{\mathrm{e}}$, and given by [5] as

$$
G_{e}=\frac{P_{r e c}}{P_{V}+P_{H}}
$$

Where $\boldsymbol{P}_{\boldsymbol{V}}$ and $\boldsymbol{P}_{\boldsymbol{H}}$ are, respectively, the mean powers of the vertically polarized (VP) and horizontally polarized (HP) radio waves received while the antenna moves over a random route in the environment. The mean power ratio of the $\boldsymbol{V P}$ and $\boldsymbol{H P}$ radio waves is defined as the cross-polarization power ratio $\left(\boldsymbol{X P R}=\boldsymbol{P}_{\boldsymbol{V}} / \boldsymbol{P}_{\boldsymbol{N}}\right)$. $\boldsymbol{X P R}$ corresponds to the cross-polarization coupling when the polarization of the transmitted radio waves is horizontal, and to the reciprocal of the cross-polarization coupling when the polarization of transmitted waves is vertical.

In general, if the complex correlation coefficient is $\rho$ and the correlation coefficient for the observed envelope is $\boldsymbol{\rho}_{\mathrm{e}}$, then $\boldsymbol{\rho}_{\mathrm{e}}$ is approximately equal to $|\boldsymbol{\rho}|^{2}$, and is given by [5] as

$$
\rho_{e} \approx|\rho|^{2}=\frac{\left|R_{12}\right|^{2}}{\sigma_{1}^{2} \sigma_{2}^{2}}=\frac{\left|\left\langle V_{1}(t) V_{2}^{*}(t)\right\rangle\right|^{2}}{\left\langle V_{1}(t) V_{1}^{*}(t)\right\rangle\left\langle V_{2}(t) V_{2}^{*}(t)\right\rangle}
$$

Where $V_{1}(t)$ and $V_{2}(t)$ are the received voltages of the two antennas diversity branches, $\boldsymbol{R}_{\mathbf{1 2}}$ is the cross-covariance of the two received voltages, and $\boldsymbol{\sigma}_{\mathbf{1}}, \boldsymbol{\sigma}_{\mathbf{2}}$ are the standard deviations of the complex envelopes of the first and second antenna. The cross covariance $\boldsymbol{R}_{\mathbf{1 2}}$ is given by the following equation

$$
R_{12}=\int_{0}^{2 \pi} \int_{0}^{\pi}\left[\begin{array}{l}
X P R \cdot E_{\theta 1}(\theta, \phi) E_{\theta 2}^{*}(\theta, \phi) P_{\theta}(\theta, \phi)+ \\
E_{\phi 1}(\theta, \phi) E_{\phi 2}^{*}(\theta, \phi) P_{\phi}(\theta, \phi)
\end{array}\right] e^{-j k x} \sin \theta d \theta d \phi
$$

where $\boldsymbol{k}$ is the wave number, and $\boldsymbol{k} \boldsymbol{x}$ is the phase difference of the angular density functions of the incident waves $P_{\theta}(\theta, \phi)$ and $P_{\phi}(\theta, \phi)$ as seen at the two antennas. The standard deviations $\boldsymbol{\sigma}_{\boldsymbol{m}}$ for the first $(\boldsymbol{m}=1)$ and the second $(\boldsymbol{m}=2)$ antennas are given by

$$
\sigma_{m}^{2}=\int_{0}^{2 \pi} \int_{0}^{\pi}\left[\begin{array}{l}
X P R \cdot E_{\theta m}(\theta, \phi) E_{\theta m}^{*}(\theta, \phi) P_{\theta}(\theta, \phi)+ \\
E_{\phi m}(\theta, \phi) E_{\phi m}^{*}(\theta, \phi) P_{\phi}(\theta, \phi)
\end{array}\right] \sin \theta d \theta d \phi
$$

Assuming that the incident waves arriving at the mobile antenna are concentrated in 
the horizontal plane and that the angular density functions of the incident waves are given by:

$$
P_{\theta}=P_{\phi}=\frac{1}{2 \pi} \delta\left(\theta-\frac{\pi}{2}\right)
$$

Substituting the incident waves of (5) into (4) and (3), and then the resultant into (2) to give the following simplified form of $\rho_{\mathrm{e}}$ as

$$
\rho_{e} \approx|\rho|^{2}=\frac{\int_{0}^{2 \pi} P_{12}\left(\frac{\pi}{2}, \phi\right) d \phi}{\left\{\int_{0}^{2 \pi} P_{11}\left(\frac{\pi}{2}, \phi\right) d \phi \times \int_{0}^{2 \pi} P_{22}\left(\frac{\pi}{2}, \phi\right) d \phi\right\}^{\frac{1}{2}}}
$$

Where $P_{m n}\left(\frac{\pi}{2}, \phi\right)=X P R \cdot E_{\theta m}\left(\frac{\pi}{2}, \phi\right) E_{\theta n}^{*}\left(\frac{\pi}{2}, \phi\right)+E_{\phi m}\left(\frac{\pi}{2}, \phi\right) E_{\phi n}^{*}\left(\frac{\pi}{2}, \phi\right)$

$\vec{E}_{m}(\theta, \phi)=\hat{\theta} E_{\theta m}(\theta, \phi)+\hat{\phi} E_{\phi m}(\theta, \phi)$ is the electric field intensity of antenna $m$ $=1,2$.

The antenna port correlation coefficient (APCC) may be used to estimate $\boldsymbol{\rho}_{\mathrm{e}}$, and is given by the normalized mutual resistance $r_{i j}$ as [3]

$$
A P C C=\frac{\operatorname{Real}\left(Z_{i j}\right)}{\operatorname{Real}\left(Z_{i i}\right)}=r_{i j}
$$

\section{ESTIMATION OF ANTENNA DIVERSITY CORRELATION CHARACTERISTICS}

In the design of antenna diversity systems, it is considered effective to apply the proposed simplified forms given by equations (6) and (7) to evaluate the correlation characteristics of antenna diversity. Starting by equation (6), which indicates clearly that as the cross polarization ratio XPR increases, the correlation coefficient $\boldsymbol{\rho}_{\mathrm{e}}$ is rapidly decreasing rendering better antenna diversity performance, so the mobile antenna MEG degradation factors XPR/(1+XPR), and $1 /(1+X P R)$ are plotted vs. XPR in Fig. 1, while Fig. 2 shows the variation of MEG vs. XPR for the reference antennas ( $\lambda / 2$ and short dipole antennas). It is valuably noticed from Fig.2 that a value of $\boldsymbol{X P R}$ = $10 \mathrm{~dB}$ is sufficient for optimum MEG mobile antenna, and hence optimum antenna diversity performance. Also, the achieved optimum MEG is constant regardless of XPR variation as far as $X P R>10$, which means that optimum antenna diversity performance may be obtained regardless of the polarization variation of the incident waves. 


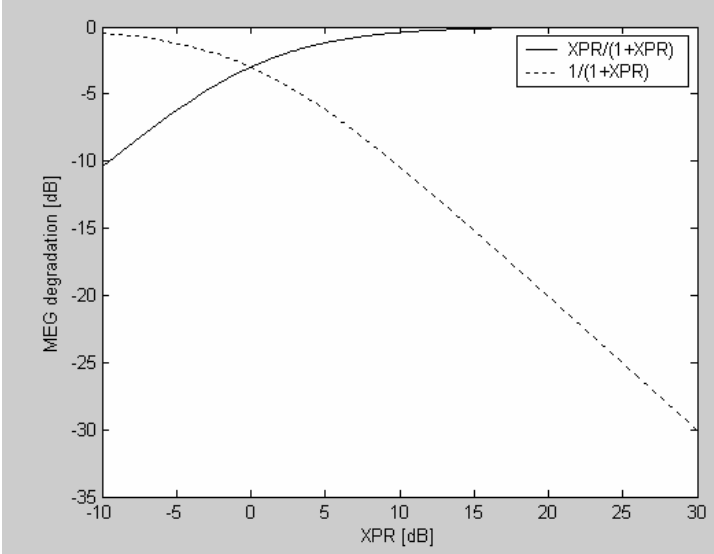

Fig.1. MEG degradation vs. XPR

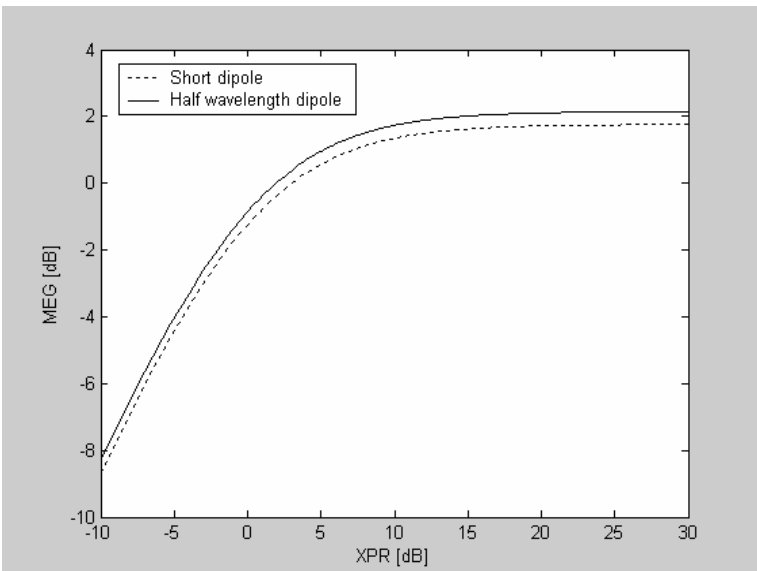

Fig.2. Dipoles MEG vs. XPR

To test the validity of equation (7) as an effective tool for evaluation of the performance of mobile antenna diversity schemes, the space diversity with two parallel half-wavelength dipoles and $\lambda / 2$ spacing, is investigated. The proposed diversity system, shown in Fig.3, is simulated using WIPL-D software package. The dipole lengths and radii are adjusted to give good matching performance within GSM900 frequency range as shown in Fig.4. Different co-polar and cross-polar pattern cuts are shown Fig.5. a, b, c, and d, from which, XPR can be estimated, and hence MEG and $\rho_{e}$ are evaluated at different sectors for good assessment of antenna diversity performance.

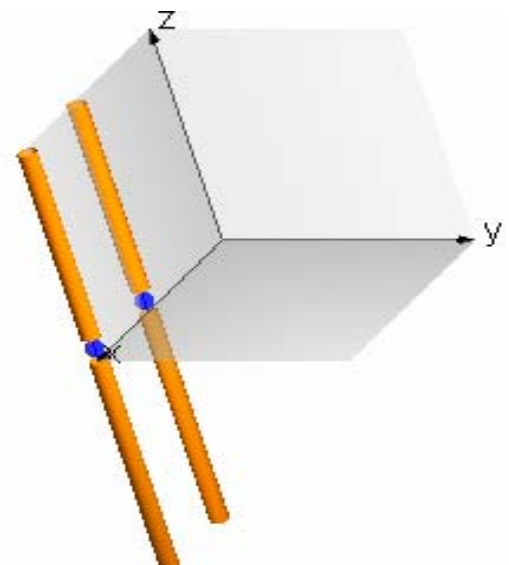

Fig.3. Two Half-wavelength dipoles

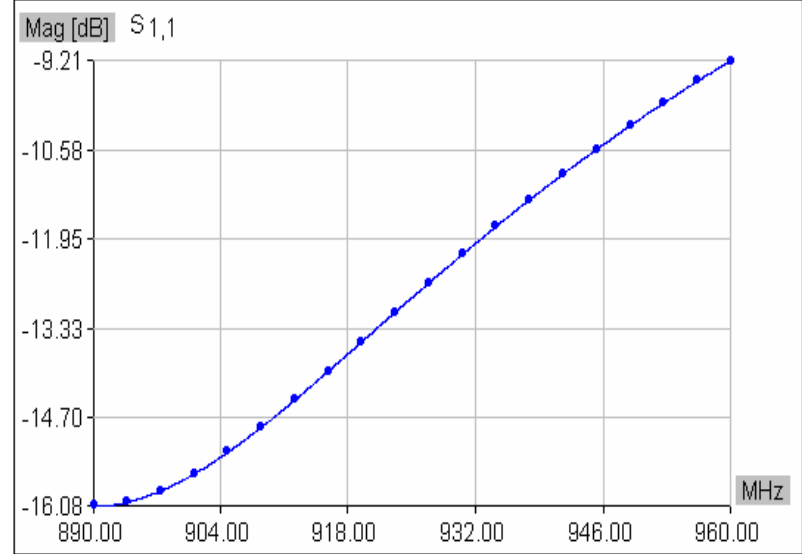

Fig.4. Antenna Return loss vs. frequency 
Eco-Gain [dB] $\theta=0 \quad 901.1 \mathrm{MHz}$

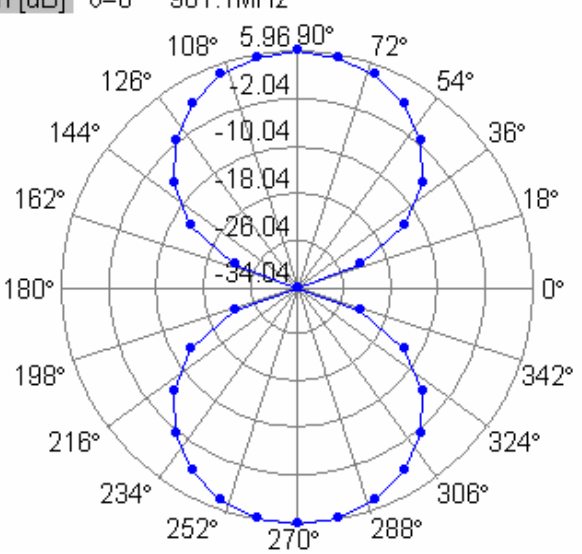

Eco-Gain [dB] $\quad \theta=100 \quad 901.1 \mathrm{MHz}$

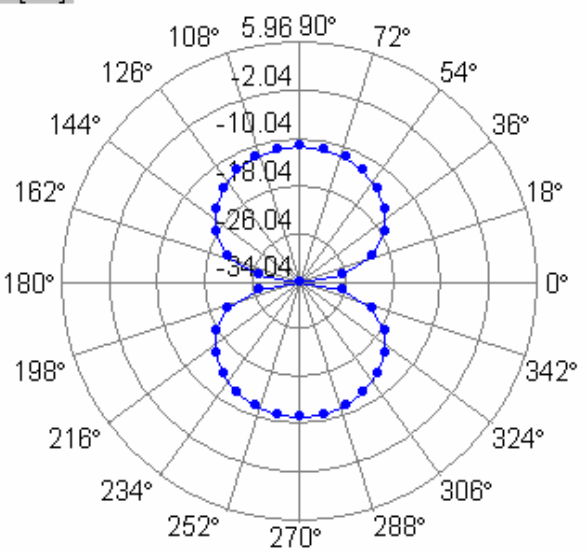

Ecr-Gain [dB] $\theta=0 \quad 901.1 \mathrm{MHz}$

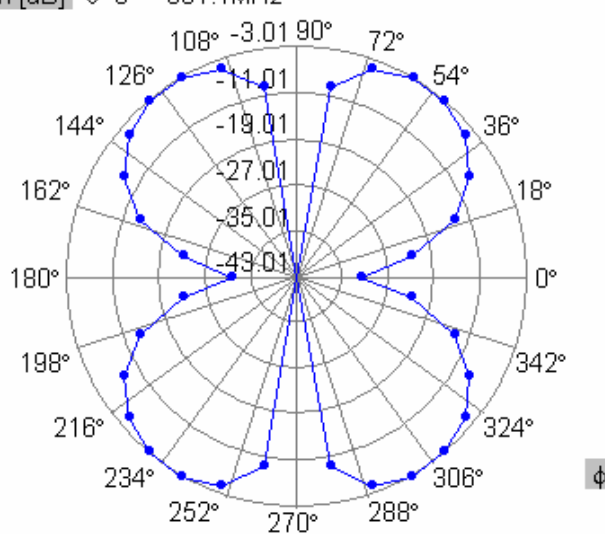

Ecr-Gain [dB] $\theta=100 \quad 901.1 \mathrm{MHz}$

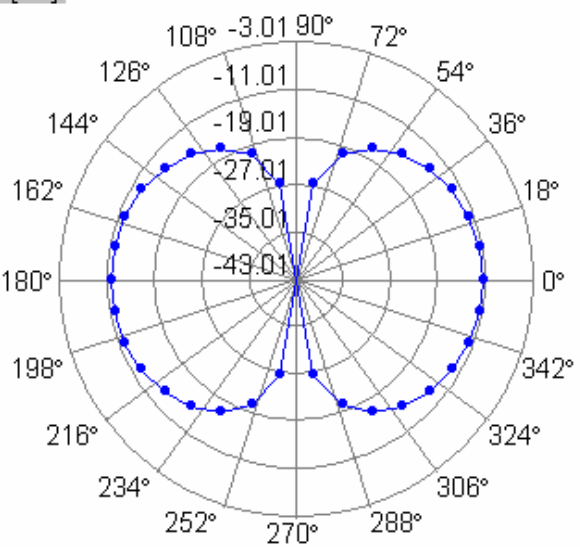

Fig.5. Different co-polar and cross-polar pattern cuts for space diversity antenna system with two parallel half-wavelength dipoles and $\lambda / 2$ spacing

The same parallel dipoles antenna diversity scheme shown in Fig.3, is considered again, but for several combinations of different dipole lengths and spacing. The mutual impedances $Z_{i j}$ are calculated numerically using the formula of [6], and the results are substituted into equation (7) to give the antenna port correlation coefficient (APCC). The results are then plotted in Figs.7 and 8, and their statistical data are tabulated in Tables 1 and 2; respectively.

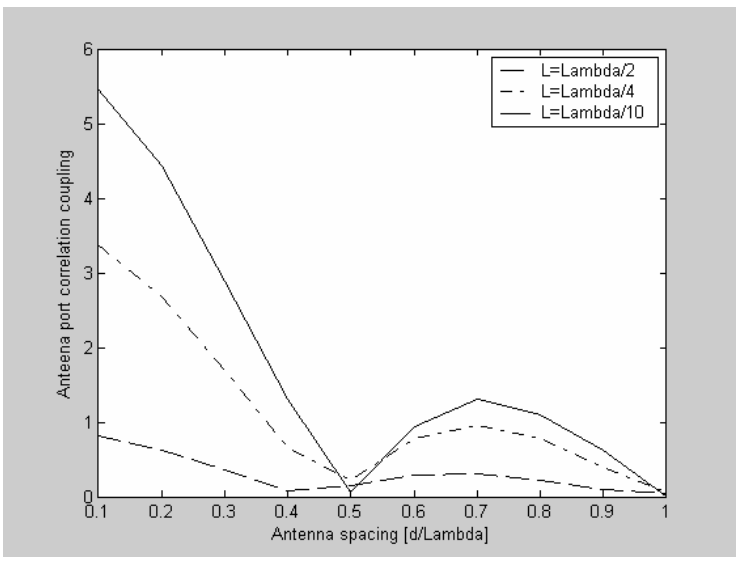

Fig.6. APCC variation vs. $d / \lambda$ for parallel dipoles with variable lengths

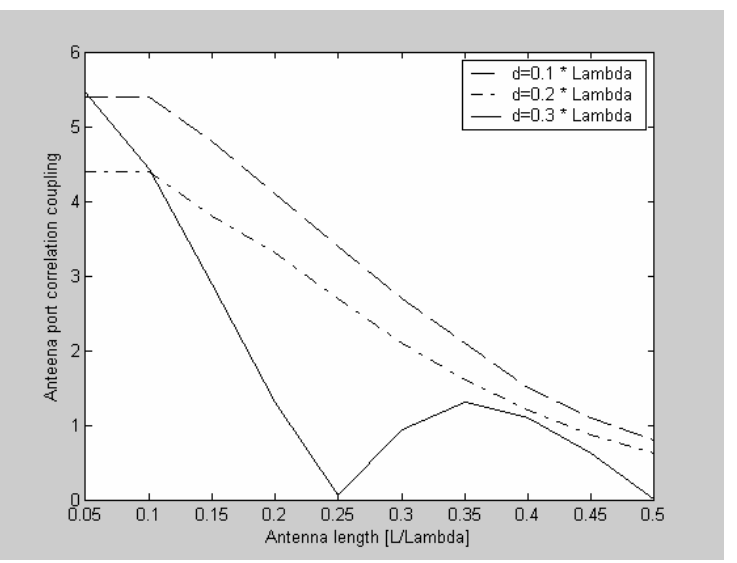

Fig. 7. APCC variation vs. dipole length for parallel dipoles with different $d / \lambda$. 


\begin{tabular}{|l|l|l|l|}
\hline $\begin{array}{l}\text { Statistical } \\
\text { APCC }\end{array}$ & $\begin{array}{l}\text { Parallel } \\
\text { Value } \\
\text { dipoles }\end{array}$ & $\lambda / 4$ & $\lambda / 10$ \\
\hline Maximum & 0.8 & 3.4 & 5.5 \\
\hline Mean & 0.3 & 1.16 & 1.8 \\
\hline Minimum & 0.05 & 0.06 & 0.02 \\
\hline
\end{tabular}

Table 1. Statistical data of Fig.7

\begin{tabular}{|l|l|l|l|}
\hline $\begin{array}{l}\text { Statistical } \\
\text { APCC } \\
\text { Value }\end{array}$ & $\begin{array}{l}\text { Parallel } \\
\text { dipoles } \\
\text { spaced by } \\
0.1 \lambda\end{array}$ & $0.2 \lambda$ & $0.3 \lambda$ \\
\hline Maximum & 5.4 & 4.4 & 5.4 \\
\hline Mean & 3.13 & 2.5 & 1.8 \\
\hline Minimum & 0.8 & 0.62 & 0.02 \\
\hline
\end{tabular}

Table 2. Statistical data of Fig. 8

For this type of space diversity with two parallel dipole antennas, Fig.6 shows that the $\lambda / 2$ dipoles exhibit the best diversity performance with correlation coefficient $\rho_{e}<1$ as the dipole spacing varies from $\lambda / 10$ up to $\lambda$, and the optimum antenna spacing for $\rho_{e}<0.5$ is achieved at spacing lies in the range $0.25 \lambda$ to $\lambda$. On the other hand, the $N / 4$ elements exhibit inferior characteristics, where element spacing should be larger than $0.35 \lambda$, with optimum spacing lies in the range $0.4<d / \lambda<0.6$. On the contrary, the $\lambda / 10$ dipole is an impractical antenna candidate for such antenna diversity schemes. Fig.7 states clearly that the practical space diversity antenna system with two parallel dipole antennas should have dipole length larger than $0.2 \lambda$ with element spacing greater than $0.3 \lambda$, given that the mobile environment is the one that is modeled by the angular density functions of the incident waves as in equation (5).

\section{CONCLUSIONS}

A proposed procedure has been developed and used to design an optimum configuration of antenna diversity by considering the characteristics of both the correlation coefficient and mobile antenna effective gain. The developed numerical code has been used to investigate the correlation characteristics of the (reference) $\boldsymbol{N} / \mathbf{2}$ and short dipole antennas. The results showed that a value of $X P R=10$ is sufficient for optimum mobile antenna effective gain, and hence optimum antenna diversity performance. These obtained XPR values are reasonable considering the measured results in urban areas are found to be between 9 and $4 \mathrm{~dB}$ [7]. Finally, several space-diversity systems with two parallel dipoles having different lengths and spacing, have been designed and their correlation characteristics have been investigated numerically and graphically. The results showed that the practical space diversity antenna system $\left(\rho_{e}<1\right)$ should have dipole length $L>0.2 \lambda$ and spacing $d>$ $0.3 \lambda$.

\section{REFERENCES}

[1] Tse, D. and Viswanath, P., "Fundamentals of Wireless Communications," Cambridge University Press, 2005. 
[2] Green, B.M and Jensen, M.A., "Diversity Performance of Dual-Antenna Handsets Near Operator Tissue," IEEE Transactions on Antennas and Propagation, Vol 48, No 7 , July 2000.

[3] Braun, C., Engblom G., and Beckman C., "Evaluation of Antenna Diversity Performance for Mobile Handsets Using 3-D Measurement Data," IEEE Transactions on Antennas and Propagation, Vol 47, No 11, November 1999.

[4] Ko, C.K. and R.D. Murch, "A Diversity Antenna for External Mounting on Wireless Handsets," IEEE Transactions on Antennas and Propagation, Vol 49, No 5, May 2001.

[5] Pahlavan, K. and Krishnamurthy, P., "Principles of Wireless Networks-A Unified Approach." Englewood Cliffs, NJ: Prentice-Hall, 2002.

[6] Stutzman, W.L. and Thiele, G.A., "Antenna Theory and Design," John Wiley \& Sons, 1981.

[7] Lee, W.C.Y., and Yeh, Y.S., "Polarization Diversity System for Mobile Radio," IEEE Trans Communications, Vol. COM-20, No.5, pp. 912-923, Oct. 1972. 